\title{
CEREBRAL COMPLICATIONS OF HYPOTENSIVE ANAESTHESIA IN A HEALTHY ADULT
}

\author{
J. B. BRIERLEY AND J. E. COOPER \\ From the Department of Neuropathology, Institute of Psychiatry, \\ the Maudsley Hospital, London
}

The employment of deliberately induced hypotension with the purpose of reducing haemorrhage or oedema is a procedure that does not yet receive the unequivocal endorsement of all anaesthetists and surgeons. The inherent risk is that of ischaemia in vital organs.

This report presents the psychiatric, neurological, and pathological sequelae of a plastic operation (mammaplasty) carried out under drug-induced hypotension in a healthy, normotensive woman of 43 years who died 23 months later. The return of consciousness was delayed, and the patient showed an organic dementia characterized by a Korsakoff psychosis and also some permanent Parkinsonian features.

\section{CASE REPORT}

The patient was a previously healthy woman aged 43 , who underwent a bilateral Beisenberger mammaplasty for cosmetic reasons. Physical examination before the operation showed no abnormalities and her blood pressure was recorded as $130 / 90 \mathrm{~mm}$. $\mathrm{Hg}$.

OPERATION Anaesthesia was induced by Pentothal $430 \mathrm{mg}$., Flaxedil $80 \mathrm{mg}$., and Arfonad $50 \mathrm{mg}$. This was followed by Pentothal $500 \mathrm{mg}$. and Flaxedil $40 \mathrm{mg}$., in split doses, plus nitrous oxide and oxygen. The patient was put into a position of about $20^{\circ}$ head-up tilt, and during the first 30 minutes a further $50 \mathrm{mg}$. of Arfonad was given in split doses.

The systolic blood pressure was kept at between 80 and $85 \mathrm{~mm}$. $\mathrm{Hg}$, and when, after about 30 minutes, a few sighing respirations were noted an immediate check showed that the systolic blood pressure was unrecordable. The head of the table was at once lowered and $15 \mathrm{mg}$. Methedrine given intravenously, which resulted in the rapid return of the blood pressure to $80 \mathrm{~mm}$. $\mathrm{Hg}$. It was estimated that the maximum duration of this episode was about three minutes. Respiration returned to normal and the operation was completed uneventfully in a total time of one hour and 55 minutes. The blood pressure was recorded as $110 / 78 \mathrm{~mm} . \mathrm{Hg}$ at the end of the operation; six hours later colour and respiration were normal and the pulse 100 a minute.

Consciousness was not regained until the end of the third post-operative week.
On the first post-operative day lumbar puncture yielded a clear fluid under a pressure of $200 \mathrm{~mm}$. of water and the blood pressure was $120 / 80 \mathrm{~mm}$. Hg. Intravenous Megimide did not affect the level of consciousness. The plantar responses were flexor.

There was no change until the sixth post-operative day when occasional flexor spasms of the spine were noted and there was more response to stimulation.

By the twelfth day, there appeared to be some fixation of the eyes on nearby objects, and, although tone was increased in the arms, the tendon reflexes were reported as normal. On the twenty-first post-operative day, the patient seemed to comprehend simple instructions and began to take food slowly by mouth.

Neurological examination at three and a half weeks showed normal fundi and equal and reactive pupils. All four limbs were spastic but some voluntary movement was present and was stronger on the right. Pin-prick was felt on both sides of the body and both plantar responses were extensor. Slow improvement continued and, five weeks after the operation, the patient began to say 'yes' and 'no' in reply to simple questions and to regain bowel and bladder control.

At six weeks. walking was possible with assistance but as speech returned it was apparent that there was disorientation and a gross memory deficit for both past and present events. Emotional lability developed with violent temper tantrums at minor frustrations.

Slow improvement was maintained, and four months after the operation, the patient knew her name and that she was in hospital, but not the date, her address, or the name of the hospital. She could converse about very simple matters but confabulated freely when asked how she spent the time. Her speech was slurred but there was no dysphasia. All limbs were hypertonic with exaggerated reflexes. She walked in a flexed manner without swinging her arms. The left side was slightly weaker than the right and no sensory loss was found. The plantar reflexes were equivocal.

At this time an E.E.G. was reported as follows: 'A low-voltage alpha rhythm at $8 \mathrm{c} / \mathrm{s}$. is disturbed in all leads by continuous slow activity at 4 to $6 \mathrm{c} / \mathrm{s}$. In addition, low voltage very slow waves at $1 \frac{1}{2} \mathrm{c} / \mathrm{s}$. are seen intermittently, most marked over both temporal regions. The record is symmetrical and no focal abnormality is seen'.

Durıng the fifth month the patient's noisiness and outbursts of temper increased and could not be controlled by chlorpromazine and led to her transfer to a psychiatric 
hospital seven and a half months after the operation. Her physical state on admission was unchanged except that the plantar responses were now flexor. The patient wandered about the ward, talking or singing to herself; she knew her name and that she was in hospital but did not know the date, her age, or the number of her children. She had a few vague memories of her childhood but had a complete loss of memory for most of her life and for all recent events, confabulating freely both spontaneously and when encouraged. Her mental state could be legitimately described as that of a Korsakoff psychosis.

An E.E.G. eight months after the operation now showed symmetrical alpha activity at 8 to $10 \mathrm{c} / \mathrm{s}$. mixed with some slower activity. No abnormal wave forms were produced by overbreathing or photic stimulation and a second record (seconal gr. 3) showed no asymmetry of barbiturate fast activity. Psychological testing gave a Wechsler-Bellevue full-scale I.Q. of 61 (performance 59, verbal 69).

There was no change in her memory, comprehension, or behaviour over the next eight months. During frequent episodes of weeping, the patient said that she was God's daughter and that she was always talking to Him. She was usually continent of faeces but incontinent of urine. Further psychological testing, seven months after the first, showed no significant difference. The Wechsler full-scale I.Q. was 58 (performance 59, verbal 68 ).

Fourteen months after the operation the patient was transferred to a long-stay psychiatric unit where she remained until her sudden death nine months later, one year and 11 months after the operation. She collapsed during a meal and died within a few minutes, the cause of death being found at necropsy to be asphyxia from inhalation of food particles.

\section{NECROPSY REPORT}

The body was that of a well-nourished female and exhibited cyanosis. There were bilateral healed submammary scars.

Food particles were seen in the upper respiratory passages. The lungs were greatly congested and oedematous.

Minimal aortic atheroma was the only significant abnormality in the cardiovascular system.

Appearances in the other organs were normal and the cause of death was asphyxia due to the inhalation of food.

BRAIN The brain had been thinly sliced in the fresh state. As far as could be judged there were no internal herniae and the basal vessels appeared thin-walled and soft, no trace of atheroma being seen. The leptomeninges were thin, stripped easily, and the subjacent cortex was smooth. There appeared to be no appreciable ventricular dilatation. The cortical ribbon was of normal thickness except in the occipital lobe where it was unusually thin on either side of the calcarine fissue and was separated from the underlying white matter by a thin grey line.

The Ammon's horns were of normal size and appearance. The amygdaloid and caudate nuclei, the putamina, and the globi pallidi also appeared normal. The thalami showed no gross abnormality. In the cerebellum there was a suggestion of narrowing of the folia, particularly in the dorsal vermis, while the white matter and the dentate nucleus appeared normal.

Microscopic Appearances Coronal blocks from frontal, temporal, parietal and occipital lobes (including the amygdaloid nucleus, corpus striatum, thalamus, and hypothalamus) as well as from the mid-brain, pons, medulla, upper cervical spinal cord, and cerebellum were embedded in celloidin. Sections were stained by the method of Nissl (using cresyl violet), of Heidenhain for myelin, Holzer for fibrous glia, and with Mallory's phosphotungstic-acid-haematoxylin, haematoxylin and eosin, and with iron haematoxylin and Van Gieson's counterstain.

Frozen sections were also stained with oil red $O$ for fat.

Appropriate stains showed no evidence of fat embolism or of thrombosis in meningeal or intracerebral vessels.

Cortical damage was present throughout the occipital lobe, being most severe on the medial aspect but sparing the calcarine cortex itself. Typically, neuronal destruction was most conspicuous in the third layer where it often took the form of a continuous but irregular pseudolaminar sclerosis. Alternatively, the cell loss was patchy and often clearly perivascular with extension into the second and the fourth layers (Fig. 1). In the floors of

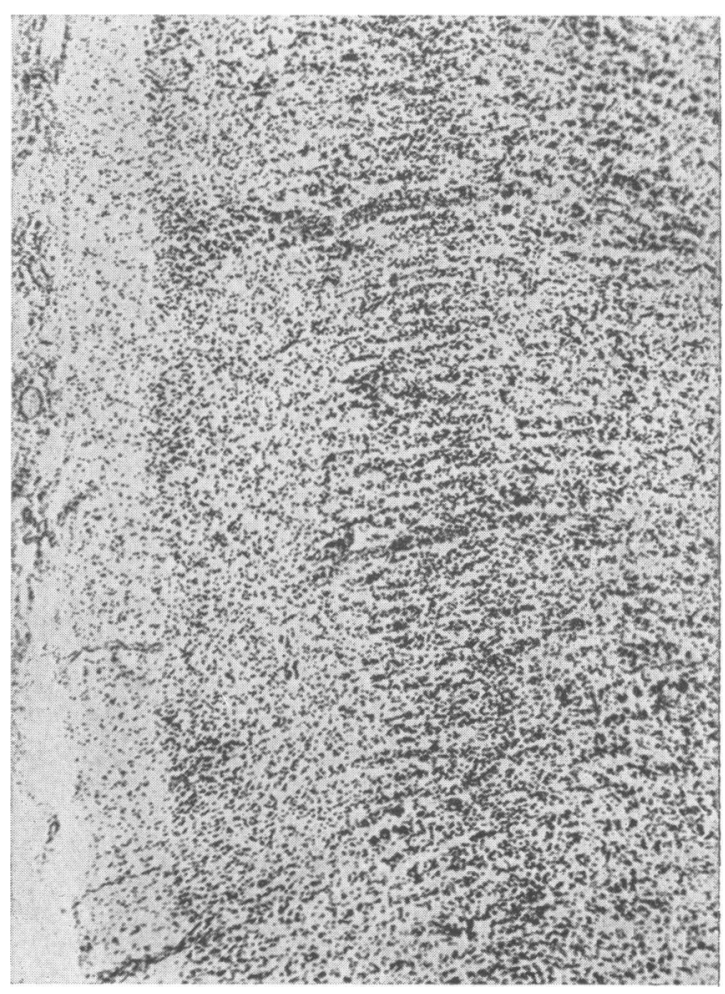

FIG. 1. Occipital cortex showing patchy neuronal destruction and gliosis (cresyl violet $\times 45$ ). 
some sulci all layers were severely involved and only a few neurones had survived.

Within the abnormal areas capillaries were only slightly prominent and the remaining neurones stained normally apart from a small number which were hyperchromatic. Ischaemic cell change was not seen. There was a moderate proliferation of astrocytes, most marked in the third layer, and also a light marginal gliosis in the molecular layer.

The central white matter of the lobe showed no obvious demyelination but there was slight pallor of myelin staining in the white cores of the gyri, particularly on the medial aspect of the lobe.

Within the cortex the radial and tangential fibres were reduced so that the bands of Baillarger were not recognizable. In the calcarine cortex the striae Gennari were visible but unusually pale. There was a slight subependymal fibrous gliosis which could be traced outwards for a variable distance into the central white matter. Apart from this, the latter contained only a few fibrous astrocytes, often in relation to blood vessels.

In the frontal lobes the appearances were essentially similar to those already described but with neuronal destruction largely confined to the third layer. The loss of intracortical myelinated fibres was striking, the bands of Baillarger and the tangential fibres having virtually disappeared.

In the temporal lobes the first temporal gyrus showed only minimal patchy thinning of neurones in the third layer, but cell loss and gliosis increased through the second and third gyri to a maximum in the lingual and fusiform gyri, while all layers were involved in the floors of the intervening sulci.

The hippocampal gyrus, including the entorhinal region, was well preserved and showed no gliosis.

In general, damage was more severe in the left hemisphere, including the white matter, which showed a marked pallor of myelin staining apart from the visual radiation which appeared normal.

In the Ammon's horns (hippocampi) there were only minor lesions. On the left there was a small wedge-like zone of cell loss in H.1 (Fig. 2) while on both sides there was some thinning of neurones in H.2, rather more marked on the left. In these abnormal areas there were a few shrunken hyperchromatic cells showing encrustations. The endfolia (H.3 to H.5) and the fascia dentata were unaffected.

No abnormalities were seen in the cortico-medial and baso-lateral portions of the amygdaloid nuclei or in the caudate nucleus, putamen, globus pallidus, or claustrum of either side.

In the thalamus, the anterior complex was almost devoid of nerve cells bilaterally and exhibited a uniformly dense astrocytic gliosis (Fig. 3). In the dorsomedial nucleus the magnocellular portion was intact but there was gross patchy neuronal loss and a uniform gliosis in the parvo-cellular portion.

There was light, patchy, and often perivascular cell loss and gliosis in the nucleus ventralis lateralis while the sensory relay nuclei (nuclei ventralis posterolateralis and posteromedialis) were intact. The nucleus lateralis dorsalis, the nucleus lateralis posterior, and the pulvinar

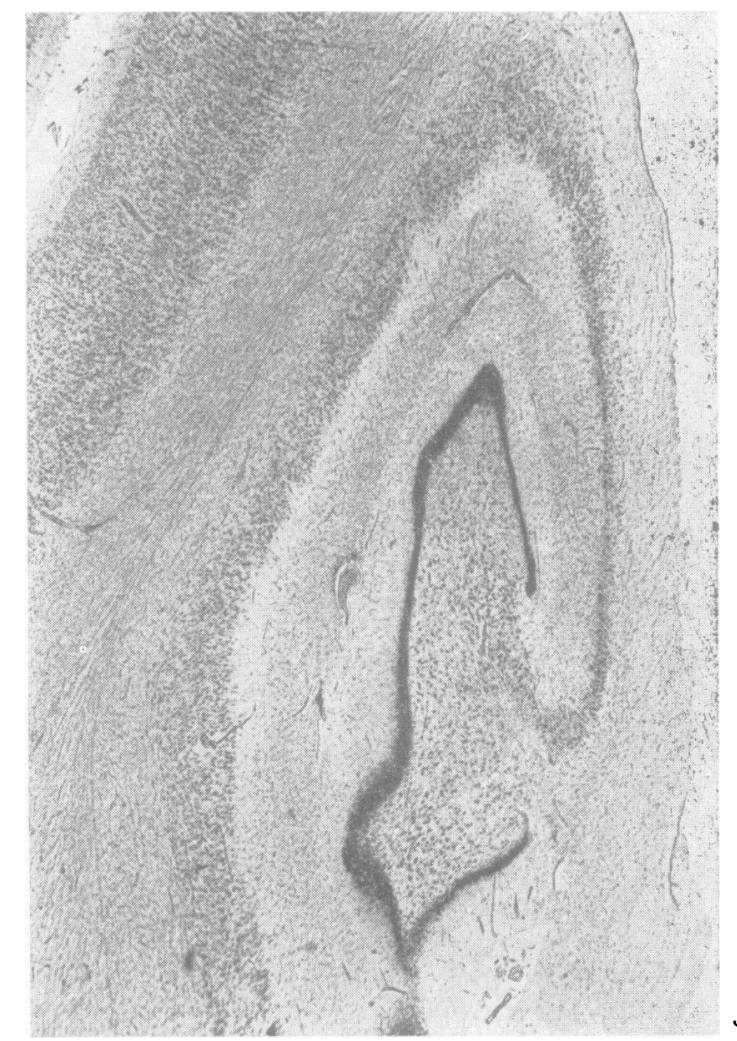

FIG. 2. Left hippocampus showing only minor areas of pyramidal cell loss in zones $H .1$ and H.2 (cresyl violet $\times 15$ ).

showed numerous small areas of neuronal loss with a marked corresponding gliosis. The medial and lateral geniculate bodies presented normal appearances.

In the subthalamic region, Forel's fields H.1 and H.2, the zona incerta, and the subthalamic nucleus were normal.

The major nuclei of the supraoptic and tuberal portions of the hypothalamus were also normal, showing no increase in capillaries or glia. Unfortunately, the mammillary bodies could not be identified in the brain as received.

In the midbrain, changes were confined to the substantia nigra. The pigmented zone was normal but in the reticular portion there was patchy cell loss and a slight gliosis.

There were no abnormalities in the grey or white matter of the pons while in the medulla there was only a slight diffuse cell loss and gliosis in the dorsal limb of the olive.

The upper cervical spinal cord was normal.

In the cerebellum there was considerable loss of Purkinje cells (Fig. 4), more marked on the superior than the inferior aspects of the hemispheres and vermis. There was a heavy proliferation of the Bergmann glia and a diffuse gliosis in the molecular layer with moderate 


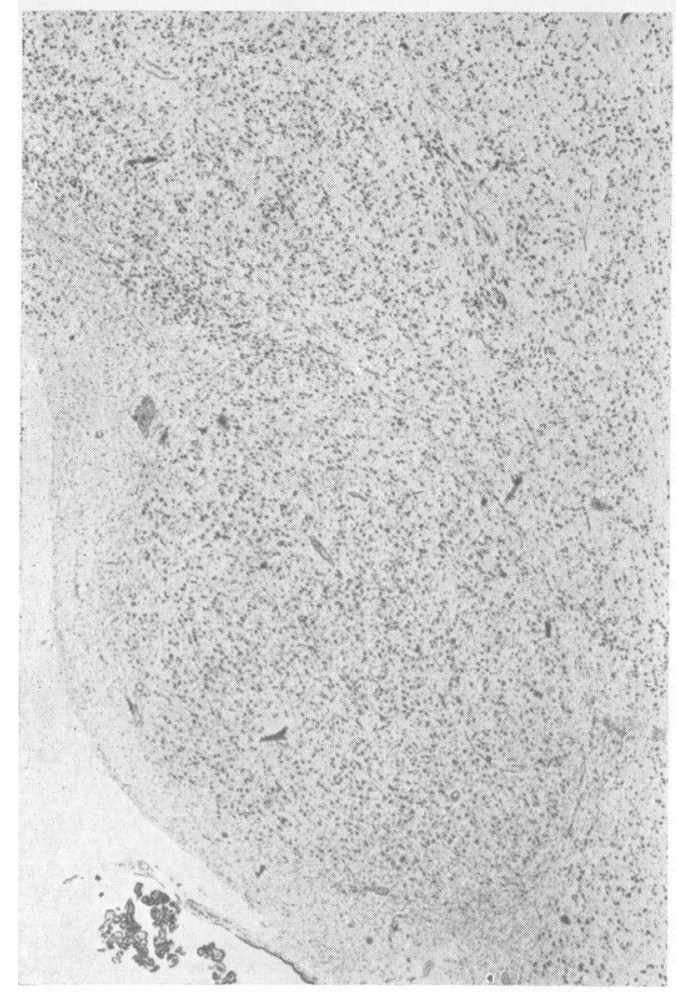

(a)

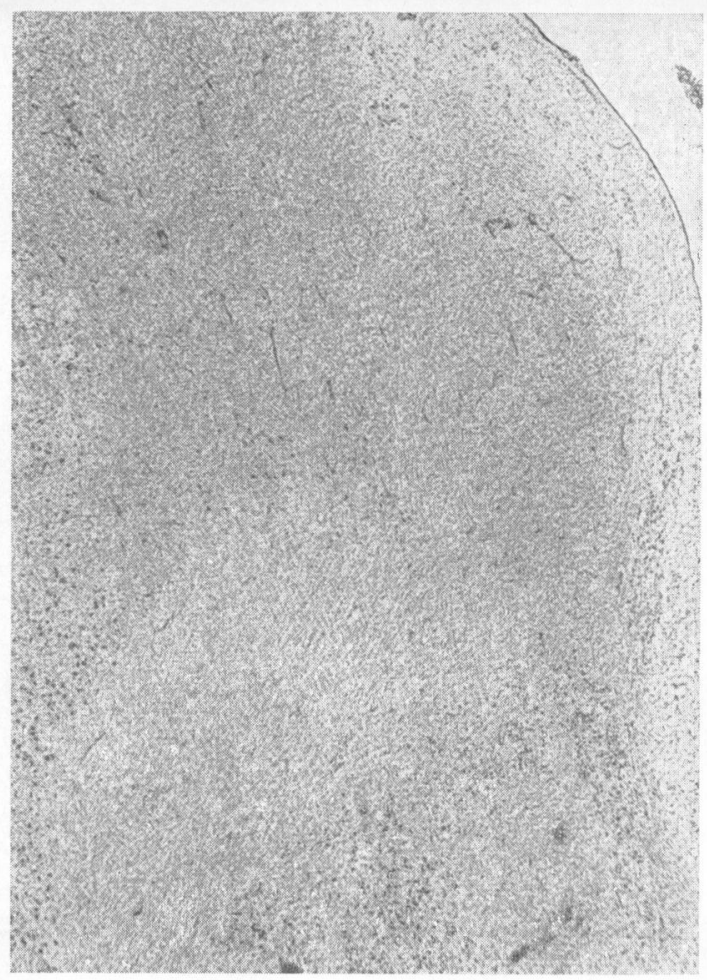

(b)

FIG. 3. (a) Normal thalamus showing anterior and upper part of dorsomedial nuclei (cresyl violet $\times 18$ ). (b) Thalamus of the case showing neuronal loss and dense gliosis in anterior complex and in parvo-cellular part of dorsomedial nucleus (cresyl violet $\times 18$ ).

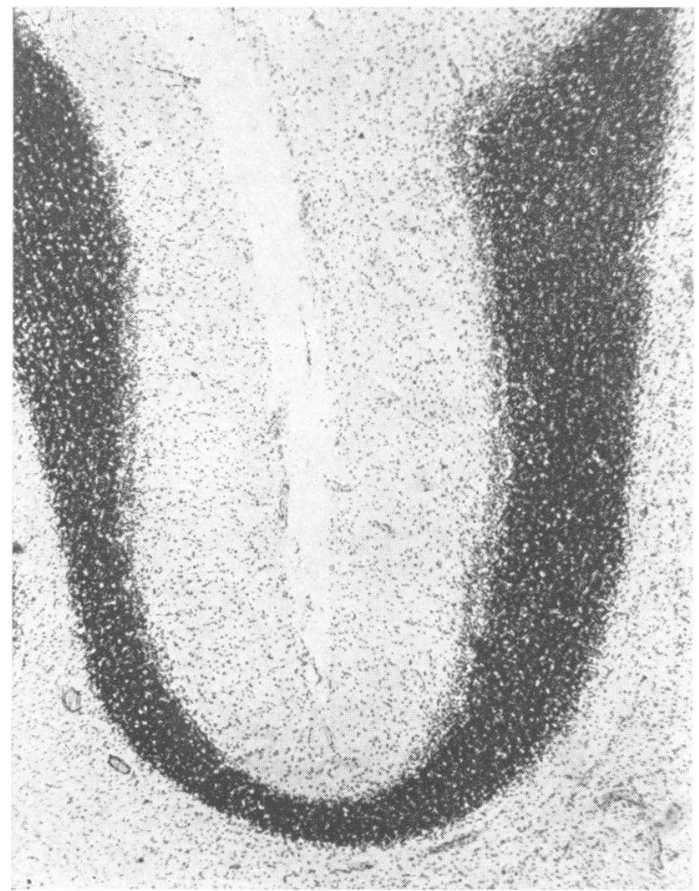

FIG. 4. Cerebellum showing almost complete loss of Purkinje cells, proliferation of Bergmann glia, and gliosis in molecular layer (cresyl violet $\times 45$ ). 
fibre formation. The granule layer was unaffected and the white matter cores of the folia were undamaged.

In the dentate nuclei there was a moderate loss of neurones and an associated light fibrous gliosis.

To sum up, cortical damage was most marked in the occipital lobe and took the form of a pseudolaminar cell loss, often with a perivascular distribution and more marked in the floors of sulci. The Ammon's horns showed only minor cell loss in zones H.1 and H.2. In the thalamus, neuronal loss was almost complete in the anterior complex and rather less in the dorsomedial and ventrolateral nuclei. The geniculate bodies were intact. There was some cell loss in the reticular zone of the substantia nigra and a marked loss of Purkinje cells in the cerebellum.

\section{DISCUSSION}

It must be concluded that, in the present case, vascular hypotension resulted in sufficient cerebral damage to produce the ensuing neuropsychiatric picture, in spite of the fact that the only cli.ical sign of anoxia during the operation was a few sighing respirations at the end of the first hour. The cerebral lesions cannot be accounted for by fat embolism and there is also no evidence of vascular pathology, whether systemic or cerebral. This would appear to be the first reported instance of delayed death following operation under hypotensive anaesthesia in which detailed clinical and pathological findings have been presented. It also appears to be the first report of a fatality since the 14 cases briefly presented by the Committee upon Deaths Associated with Anaesthesia in 1953.

While the death rate from hypotensive anaesthesia in Great Britain was stated to be 1 in 459 by Hampton and Little (1953), it is probable that the incidence of complications, particularly neurological and psychiatric, is greater. Bedford (1955) has drawn attention to the existence of dementia, particularly in minor degrees, as a complication of anaesthesia in the elderly (with hypotension among the aetiological factors), but the present case indicates that, under certain conditions, the risk exists for the substantially younger, healthy adult.

The overall cerebral cortical pathology is clearly that associated with some form of anoxia. Thus the neuronal loss is of laminar or pseudo-laminar type, centred mainly on the third layer and is greatest in the depths of sulci. In the cerebellum also, there is a typical loss of Purkinje cells and proliferation of the Bergmann glia.

An unusual feature is the very minor extent of the damage in the pyramidal cell layer of the Ammon's horns. The thalamic lesions, on the other hand, are of a severity at least equal to those in the occipital cortex. In view of the relatively slight involvement of the frontal and cingulate areas, these thalamic lesions must be attributed to a primary 'anoxic' rather than a retrograde pathogenesis and are moreover of a type often encountered in anoxia.

In view of the known episode of hypotension during the operation, the resulting 'anoxia' must te regarded as of 'ischaemic' type and it is of interest to compare the lesions in this case with those due to other types of anoxia.

All degrees of laminar and pseudo-laminar cortical necrosis can be encountered in 'ischaemic' anoxia (Blackwood, 1958), and are seen in the present case but also in virtually all other types of anoxia.

Focal and sometimes perivascular cortical lesions also occur in cases surviving cardiac arrest (Meyer, 1956), respiratory arrest (Courville, 1954; Meyer, 1949), carbon monoxide poisoning, and hypoglycaemia (Meyer, 1958). Sharply defined perivascular lesions have been reported by Ingraham and Bering (1959) in the cerebral cortex of monkeys rendered hypotensive with Arfonad, so it may well be that after hypotension cortical lesions are more clearly perivascular than after anoxic anoxia.

With regard to the pattern of lesion distribution, Scholz (1952) has suggested that after hypoxaemia (anoxic anoxia), cortical lesions are minimal, the brunt of the damage falling upon Ammon's horn and the diencephalon. Ischaemia and oligaemia, on the other hand, are held to produce the more severe cortical lesions with but little subcortical involvement. In the present case, the combination of diffuse cortical and severe thalamic lesions does not support these conclusions. The view is expressed that these histopathological findings do not constitute an entity distinct from those due to other types of anoxia.

The presence of a typical Korsakoff psychosis during life is of interest in view of the absence of its usual pathological substrate, the 'acute superior haemorrhagic polio-encephalitis' of Wernicke (1882). Thus there was no evidence of capillary proliferation, gliosis, or cell loss in the supraoptic or paraventricular hypothalamic nuclei, in the periaqueductal grey matter, or in the floor of the fourth ventricle. The absence of the mammillary bodies was unfortunate in view of the fact that Rémy (1942) has shown that a lesion confined to these structures can give rise to a typical Korsakoff psychosis. The pathological substrate of the defect of memory cannot therefore be fruitfully discussed in this case.

HYPOTENSION AND HEAD-UP TILT In the proved absence of any vascular pathology (including fat embolism), whether systemic or cerebral, it is only hypotension, in some degree, that merits discussion in relation to the neuropsychiatric complications in this case. 
As far as the brain is concerned, the existing level of drug-induced hypotension will have been increased by the maintenance of a $20^{\circ}$ head-up tilt of the table during an operation lasting one hour and 55 minutes. A further factor probably due to this head-up tilt was the disappearance of a recordable blood pressure for about three minutes, half an hour after the start of the operation.

That hypotension combined with head-up tilt is not without risk has often been emphasized, and Davison $(1952,1953)$, stressing the risks to the brain of head-up tilt, has urged that apart from rare and carefully selected cases, the methonium drugs should only be used with the patient supine and then only to produce a moderate fall in blood pressure.

The Committee upon Deaths Associated with Anaesthesia briefly reported 14 deaths associated with hypotensive anaesthesia. In the majority, survival was only a matter of hours but three patients lived for two, three, and five days. In one of these there was cerebral infarction and gross cerebral atheroma and in another 'gross cerebral degeneration'. Further clinical and pathological details of these cases have not been published.

Hampton and Little (1953) on the basis of a questionnaire sent to American and British anaesthetists, analysed the complications associated with 'controlled hypotension'. In a series of 27,930 cases the commonest cause of death was cerebral thrombosis and anoxia which together accounted for 18 $(20 \%)$ deaths. It was also shown that a systolic blood pressure of less than $80 \mathrm{~mm}$. $\mathrm{Hg}$ resulted in a significantly greater incidence of all types of complication, but in this series the position of the operating table was not recorded.

In the conscious subject, Bromage (1953) demonstrated the effects of head-up tilt upon the E.E.G. when hypotension was induced with pentamethonium iodide. In one normotensive case, a blood pressure of $80 / 60 \mathrm{~mm}$. $\mathrm{Hg}$ together with a head-up tilt of $45^{\circ}$ produced no E.E.G. or other changes while in the second normotensive subject a $45^{\circ}$ tilt produced loss of consciousness, clonic convulsions of the face and arms. and abolition of the alpha rhythm.

Additional evidence of abnormal cerebral metabolism when low blood pressure is combined with head-up tilt was provided by Nilsson (1953) using the flicker fusion frequency test in a post-operative study of 15 patients who had been rendered hypotensive with hexamethonium. Positive results indicative of cerebral injury were obtained in six, five of whom had been subjected to a head-up tilt of 10 to $20^{\circ}$.

In their series of 14 anaesthetized patients, Van Bergen, Buckley, French, Dobkin, and Brown (1954) carried out a detailed study of the physiological alterations associated with hexamethonium. They noted that not only does general anaesthesia enhance the effects of the drug but that a head-up tilt resulted in a further fall in systolic blood pressure of $22.6 \%$ for $10^{\circ}$ and $39.7 \%$ for $15^{\circ}$. Further, in neurosurgical cases in a $15^{\circ}$ Fowler's position 'on several occasions severe cerebralcyanosis developed although the toenail beds remained pink'. It was stressed that when vital organs are among the elevated portions ... it is important that an accurate knowledge of the intra-arterial pressure of the vessels supplying the organ should be known'.

The flicker fusion frequency test was again employed by Berg, Nilsson, and Vinnars (1957) in a study of 73 surgical patients subjected to hypotensive anaesthesia. Thirty-eight gave positive results persisting for at least six days while the controls without hypotension were all negative. Of the 38 , 10 remained positive for more than six days and of these eight were operated upon in the head-up position. These authors concluded that it was obvious that in patients with a blood pressure below $80 \mathrm{~mm} . \mathrm{Hg}$ the induction of hypotension involves greater risks if the head end of the table is elevated'.

This brief review of the complications and fatalities associated with hypotensive anaesthesia, particularly in the presence of head-up tilt, must lead to an enquiry into the alterations in cerebral circulation and metabolism that occur under these conditions.

While there is now considerable information on the cerebral blood flow, vascular resistance, and oxygen consumption during hypotension in the supine position, there is little that concerns these variables when the head is raised.

Little (1956) has stated that 'certain postures threaten ischaemia to vital organs, particularly the brain, because induced hypotension and gravity may combine to lower the local blood pressure (as quite distinct from the blood pressure at heart level) below that necessary to fulfil the requirements of cerebral metabolism'. Enderby (1954) calculated that with a $30^{\circ}$ head-up tilt and a cardiac systolic blood pressure of $60 \mathrm{~mm}$. $\mathrm{Hg}$ the cerebral blood pressure would be only $30 \mathrm{~mm}$. $\mathrm{Hg}$.

Stephen, Woodhall, Odom, Reynolds, BourgeoisGavardin, Martin, and Bloor (1956), using a strain gauge, measured the pressure in cerebral arteries exposed during neurosurgical procedures. It was noted that with a head-up tilt of 10 to $20^{\circ}$ cortical blood pressure fell to between 1 and $35 \mathrm{~mm}$. $\mathrm{Hg}$ which '... . could bring about a localized arrest of circulation with resulting hypoxia of cerebral tissues'.

The conclusion of Davison (1958), that ' . . by suitable positioning of the subject any part can be deprived of its blood', seems fully justified by the evidence that has been reviewed. 
Burton (1958) has shown that blood flow within a vessel may cease although some blood pressure (the critical closing pressure) remains. At this point the elastic tension in the vessel wall exceeds the hydrodynamic pressure of the blood. In respect of cerebral blood flow, it is generally agreed that this is either normal or only moderately reduced under hypotension in the supine position. As a result of the associated vasodilatation, the fall in peripheral vascular resistance, the increased extraction of oxygen by the brain and its reduced oxygen requirements due to anaesthesia, cerebral metabolism remains within normal limits (Saunders, 1954; Stone, Mackrell, and Wechsler, 1954; 1955; Hughes, 1955; Little, 1956).

In the head-up position, however, there is no agreement that these several changes can maintain adequate blood flow and oxygen supply, and the present case is almost certainly an instance of their failure to do so in spite of the correct fulfilment of the normally accepted surgical and anaesthetic criteria of safety.

Although a majority of hypotensive operations are without clinical evidence of harmful effects, this case represents an endorsement of the warnings already quoted to the effect that hypotension, combined with head-up tilt of the operating table, can be a hazardous procedure in a healthy subject.

\section{SUMMARY}

The case of a healthy normotensive woman of 43 years subjected to a cosmetic operation under hypotensive anaesthesia is reported.

A head-up tilt of $20^{\circ}$ was maintained during an operation period of one hour 55 minutes.

Return of consciousness was delayed and was followed by an organic dementia persisting until death one year and 11 months later.

The brain exhibited diffuse lesions of anoxic type.

This investigation has been carried out while one of us (J.B.B.) was in receipt of a research grant from the
National Spastics Society to study the pathogenesis of cerebral anoxic lesions.

The authors are indebted to Professor A. Meyer, Professor P. Daniel, and Dr. J. A. N. Corsellis for much valuable advice and criticism.

\section{REFERENCES}

Bedford, P. D. (1955). Lancet, 2, 259.

Berg, O., Nilsson, E., and Vinnars, E. (1957). Brit. J. Anaesth., 29, 146.

Blackwood, W. (1958). Vascular Disease of the Central Nervous System. In Neuropathology, ed. J. G. Greenfield, Ch. 2. Arnold, London.

Bromage, P. R. (1953). Proc. roy. Soc. Med., 46, 919.

Burton, A. C. (1958). Interrelation of Physical and Physiological Factors. In Factors Regulating Blood Flow: Proc. 3rd Conf, Microcirculatory Physiology and Pathology, Milwaukee. 1956, ed. Fulton, G. P., and Zweifach, B., p. 3. American Physiological Society, Washington, D.C.

Committee upon Deaths Associated with Anaesthesia (1953). Anaesthesia, 8, 263.

Courville, C. B. (1954). Bull. Los Angeles neurol. Soc., 19, 142.

Davison, M. H. A. (1952). Anaesthesia, 7, 116.

- (1953). Ibid, 8, 255.

- (1958). Brit. med. Bull., 14, 52.

Enderby, G. E. H. (1954). Lancet, 1, 185.

Hampton, L. J., and Little, D. M. (1953). A.M.4. Arch. Surg., 67, 549.

Hughes, G. (1955). Brit. J. Anaesth., 27, 229.

Ingraham, F. D., and Bering, E. A. (1959). Safe limits of hypothermia and hypotension. Progress report. U.S. Public $O$ Health Service. Boston.

Little, D. M. (1956). 'Controlled Hypotension' in Anesthesia and Surgery. Thomas, Springfield, Illinois.

Meyer, A. (1949). Unpublished case.

- (1956). Proc. roy. Soc. Med., 49, 619.

(1958). Anoxias Intoxications and Metabolic Disorders. In Neuropathology, ed. J. G. Greenfield, Ch. 4. Arnold, London.

Nilsson, E. (1953). Brit. J. Anaesth., 25, 24.

Rémy, M. (1942). Mschr. Psychiat. Neurol., 106, 128.

Saunders, J. W. (1954). Lancet, 1, 1156.

Scholz, W. (1952). Proc. 1st Int. Congr. Neuropath., Rome, 1952. Vol. 1, p. 321.

Stephen, C. R., Woodhall, B., Odom, G. L., Reynolds, D., BourgeoisGavardin, M., Martin, R. C., and Bloor, B. M. (1956). Ann. Surg., 143, 143.

Stone, H. H., Mackrell, T. N., and Wechsler, R. L. (1954). Amer. J. med. Sci., 228, 112.

$-, \ldots,-$ (1955). Anesthesiology, 16, 168.

Van Bergen, F. H., Buckley, J. J., French, L. A., Dobkin, A. B., and Brown, I. A. (1954). Anesthesiology, 15, 507.

Wernicke, C. (1882). Die acute hammorhagische Polioencephalitis superior. In Lehrbuch der Gehirnkrankheiten fur Aertze und Studirende, Vol. 2, p. 229. Fischer, Berlin. 